\title{
Tunable Aharonov-Anandan Phase in Transport Through Mesoscopic Hole Rings
}

\author{
M. Pletyukhov ${ }^{1,2}$ and U. Zülicke ${ }^{1,3}$ \\ ${ }^{1}$ Institut für Theoretische Festkörperphysik and DFG Center for Functional \\ Nanostructures, Universität Karlsruhe, D-76128 Karlsruhe, Germany \\ ${ }^{2}$ Institut für Theoretische Physik A, RWTH Aachen, D-52056 Aachen, Germany* \\ ${ }^{3}$ Institute of Fundamental Sciences and MacDiarmid Institute for Advanced Materials and Nanotechnology, \\ Massey University, Private Bag 11 222, Palmerston North, New Zealand
}

(Dated: April 18, 2008)

\begin{abstract}
We present a theoretical study of spin-3/2 hole transport through mesoscopic rings, based on the spherical Luttinger model. The quasi-one-dimensional ring is created in a symmetric two-dimensional quantum well by a singular-oscillator potential for the radial in-plane coordinate. The quantum-interference contribution to the two-terminal ring conductance exhibits an energy-dependent Aharonov-Anandan phase, even though Rashba and Dresselhaus spin splittings are absent. Instead, confinement-induced heavy-hole-light-hole mixing is found to be the origin of this phase, which has ramifications for magneto-transport measurements in gated hole rings.
\end{abstract}

PACS numbers: 73.21.-b, 73.23.Ad, 85.35.Ds, 03.65.Vf

Introduction. Geometric quantum phases continue to be the subject of great interest because they rather elegantly elucidate quite complex fundamental microscopic properties $\frac{1}{\underline{1}}$ The well-known Aharonov-Bohm ${ }^{2}$ and Aharonov-Casher ${ }^{3}$ effects are pertinent examples, as is the Berry phase ${ }^{4}$ acquired by a quantum system during adiabatic cyclical variation of an external parameter. The more general concept of the AharonovAnandan phase $\mathrm{e}^{\frac{5}{2}}$ arising in the cyclical evolution of a quantum state was later shown to subsume the above-mentioned three phenomena as special cases. Recent progress in our understanding of geometric phases has been spurred by numerous experimental and theoretical studies. $\underline{6}$

Modern nanofabrication techniques have made it possible to study quantum interference, and thus geometric quantum phases, in mesoscopic electronic devices. $\stackrel{7}{?}$ The first theoretical suggestions $s^{\frac{8}{-}}$ and experimental realizations ${ }^{9}$ of electronic quantum interference devices were Aharonov-Bohm interferometers. Subsequent theoretical studies predicted electronic signatures of Berry phases 10 and the Aharonov-Casher effect $11,12,13$ Inspired by possible applications in the burgeoning field of spintronics $\stackrel{14}{4}$ recent experimental efforts have been devoted to observing tunable spin-related geometric phases in semiconductor rings subject to strong spin-orbit coupling $, 15,16$ In several of these experiments, $\underline{\underline{16}}$ charge carriers moving through the ring structure are characterized by an intrinsic (spin) angular momentum equal to $3 / 2$. This is due to the fact that states in the uppermost valence band of typical semiconductors originate from p-like atomic orbitals of the mate- rial's constituent elements $\stackrel{17}{ }$ The same is true for conductionband states in $\mathrm{HgCdTe}$ quantum wells because of a band inversion. Here we provide a careful study of the complexities arising from the spin- $3 / 2$ character of charge carriers confined in a mesoscopic ring. We identify a nontrivial part of the Aharonov-Anandan (AA) phase that enters the interference contribution to the two-terminal ring conductance. The energy dependence of this geometric phase will result in a continuous shift of magneto-conductance (Aharonov-Bohm) oscillations as a function of carrier density in the ring, e.g., when a gate voltage is applied. We trace the origin of the anomalous AA phase to a confinement-induced coupling between heavy-hole $(\mathrm{HH})$ and light-hole (LH) states ${ }^{18}$ that is ever-present and unrelated to (Rashba or Dresselhaus) spin splitting due to (structural or bulk) inversion asymmetry $\stackrel{17}{\underline{1}}$ Our analysis provides a framework for interpreting numerical results 19 and complements previous analytical calculations $\frac{13}{\underline{3}}$ where $\mathrm{HH}-\mathrm{LH}$ mixing was neglected.

Below we describe our theoretical model for hole rings. Readers not interested in mathematical details could skip to the end of this part where results for the lowest ring subbands are presented. We then analyse the emerging energydependent AA phase and, in our concluding discussion, address implications for experiments.

Theoretical model for a mesoscopic hole ring. We use the Luttinger model $^{20}$ in spherical approximation ${ }^{21}$ to describe electronic states in the upper-most bulk valence band. In atomic units where $\hbar=m_{0}=1$, it reads

$$
\begin{aligned}
\mathcal{H}_{\mathrm{s}}=\left(\frac{\gamma_{1}}{2}+\frac{\gamma_{\mathrm{s}}}{2}\left[\hat{J}_{z}^{2}-\frac{5}{4}\right]\right) \hat{k}_{\perp}^{2}-\frac{\gamma_{\mathrm{s}}}{2}\left(\hat{k}_{-}^{2} \hat{J}_{+}^{2}+\hat{k}_{+}^{2} \hat{J}_{-}^{2}\right)+\left(\frac{\gamma_{1}}{2}-\gamma_{\mathrm{s}}\left[\hat{J}_{z}^{2}-\frac{5}{4}\right]\right) \hat{k}_{z}^{2} \\
\\
-\sqrt{2} \gamma_{\mathrm{s}}\left(\left\{\hat{k}_{z}, \hat{k}_{-}\right\}\left\{\hat{J}_{z}, \hat{J}_{+}\right\}+\left\{\hat{k}_{z}, \hat{k}_{+}\right\}\left\{\hat{J}_{z}, \hat{J}_{-}\right\}\right) .
\end{aligned}
$$

$m_{0}$ denotes the vacuum electron mass, $\gamma_{\mathrm{s}}=\left(3 \gamma_{3}+2 \gamma_{2}\right) / 5$ in terms of Luttinger parameters,, $20 \hat{\mathbf{k}}$ and $\hat{\mathbf{J}}$ are vector oper- 
ators of kinetic linear and spin-3/2 angular momentum, respectively, and we used the abbreviations $\hat{\mathbf{k}}_{\perp}=\left(\hat{k}_{x}, \hat{k}_{y}\right)$, $\hat{k}_{ \pm}=\hat{k}_{x} \pm i \hat{k}_{y}$, and $\hat{J}_{ \pm}=\left(\hat{J}_{x} \pm i \hat{J}_{y}\right) / \sqrt{2}$. The symbol $\{A, B\}$ stands for the anticommutator $(A B+B A) / 2$. Introducing a quantum-well confinement in the growth $(z)$ direction, two-dimensional (2D) subbands are formed. Here we will focus on the situation where only the lowest $2 \mathrm{D}$ quantumwell bound state matters. To be specific, we assume a symmetric hard-wall confinement with 2D quantum-well width $d$ and simply replace operators $\hat{k}_{z}^{2}$ and $\hat{k}_{z}$ by their respective expectation values $\pi^{2} / d^{2}$ and $0 \stackrel{22}{\underline{2}}$ Using polar coordinates for in-plane motion, we obtain $\mathcal{H}_{\mathrm{s}}^{(2 \mathrm{D})}=\mathcal{H}_{\mathrm{sb}}+\mathcal{H}_{\perp}^{(2 \mathrm{D})}$, where

$$
\mathcal{H}_{\mathrm{sb}}=\left(\frac{\gamma_{1}}{2}-\gamma_{\mathrm{s}}\left[\hat{J}_{z}^{2}-\frac{5}{4}\right]\right)\left(\frac{\pi}{d}\right)^{2}
$$

arises from the quantised motion in $z$ direction, and the inplane motion of holes is governed by the part

$$
\mathcal{H}_{\perp}^{(2 \mathrm{D})}=\left(\frac{\gamma_{1}}{2}+\frac{\gamma_{\mathrm{s}}}{2}\left[\hat{J}_{z}^{2}-\frac{5}{4}\right]\right)\left\{-\partial_{r}^{2}-\frac{\partial_{r}}{r}+\frac{\hat{L}_{z}^{2}}{r^{2}}\right\}-\frac{\gamma_{\mathrm{s}}}{2}\left(\hat{J}_{+}^{2}\left[-i L_{-}\left\{\partial_{r}+\frac{\hat{L}_{z}}{r}\right\}\right]^{2}+\hat{J}_{-}^{2}\left[-i L_{+}\left\{\partial_{r}-\frac{\hat{L}_{z}}{r}\right\}\right]^{2}\right) .
$$

$\hat{L}_{z}=-i \partial_{\varphi}$ is the in-plane orbital angular momentum, and $L_{ \pm}=\mathrm{e}^{ \pm i \varphi}$. The Hamiltonian (2b) commutes with $\hat{M}_{z}=$ $\hat{L}_{z}+\hat{J}_{z}$; hence its eigenstates can be labelled by those of $\hat{M}_{z}$. To enable further analytic progress, we eliminate the $\varphi$ dependence in off-diagonal matrix elements by the transformation $\tilde{\mathcal{H}}=\mathrm{e}^{i \hat{J}_{z} \varphi} \mathcal{H} \mathrm{e}^{-i \hat{J}_{z} \varphi}$. Due to space limitations, the straightforwardly obtained expression for the transformed Hamiltonian is omitted here.

The quasi-onedimensional in-plane ring confinement is modeled by the singular-oscillator potential ${ }^{23}$

$$
V_{\perp}(r)=\frac{\omega^{2}}{2}\left(r-\frac{R^{2}}{r}\right)^{2},
$$

which was employed before to study mesoscopic electron rings. ${ }^{24}$ In the following, the energy scale $E_{0}=$ $\pi^{2} \gamma_{1} \hbar^{2} /\left(2 m_{0} d^{2}\right)$ associated with the 2D quantum-well con- finement will serve as our energy unit. We also introduce the parameter $\bar{\gamma}=\gamma_{\mathrm{s}} / \gamma_{1}$ that measures the strength of spinorbit coupling in the valence band, the length scale $\ell_{\omega}=$ $\sqrt{\sqrt{\gamma_{1}} \hbar /\left(m_{0} \omega\right)}$ associated with the ring confinement, $\lambda_{R}=$ $\left(R / \ell_{\omega}\right)^{2}$ and $\lambda_{d}=\left(2 d /\left[\pi \ell_{\omega}\right]\right)^{2}$ representing ring radius and quantum-well width in units of the effective ring width, and the operators $\hat{\Gamma}=1+\bar{\gamma}\left[\hat{J}_{z}^{2}-(5 / 4)\right]$ and $\hat{\varrho}=r /\left(\ell_{\omega} \hat{\Gamma}^{1 / 4}\right)$ that quantify a HH-LH splitting. With these conventions, the Hamiltonian of a mesoscopic hole ring is $H \equiv\left(\tilde{\mathcal{H}}_{\mathrm{s}}^{(2 \mathrm{D})}+\right.$ $\left.V_{\perp}\right) / E_{0}=H_{\mathrm{qw}}+H_{\mathrm{rg}}$, where

$$
H_{\mathrm{qw}}=1-2 \bar{\gamma}\left[\hat{J}_{z}^{2}-\frac{5}{4}\right]
$$

arises from the HH-LH splitting in the quantum-well bound state, and the in-plane motion is governed by

$$
H_{\mathrm{rg}}=\frac{\lambda_{d}}{4} \hat{\Gamma}^{\frac{1}{2}}\left(-\partial_{\hat{\varrho}}^{2}-\frac{\partial_{\hat{\varrho}}}{\hat{\varrho}}+\frac{\hat{\tilde{L}}_{z}^{2}}{\hat{\varrho}^{2}}+\left[\hat{\varrho}-\frac{\lambda_{R} \hat{\Gamma}^{-\frac{1}{2}}}{\hat{\varrho}}\right]^{2}\right)+\frac{\lambda_{d}}{4} \bar{\gamma}\left\{\left(\hat{J}_{+} \hat{\Gamma}^{-\frac{1}{4}}\left[\partial_{\hat{\varrho}}+\frac{\hat{\tilde{L}}_{z}}{\hat{\varrho}}\right]\right)^{2}+\left(\hat{J}_{-} \hat{\Gamma}^{-\frac{1}{4}}\left[\partial_{\hat{\varrho}}-\frac{\hat{\tilde{L}}_{z}}{\hat{\varrho}}\right]\right)^{2}\right\} .
$$

Equation (4b) suggests the wave-function ansatz

$$
\psi_{n, m}(r, \varphi)=\mathrm{e}^{i m \varphi}\left(\begin{array}{c}
a_{n, m} \psi_{n, m}^{(3 / 2)}(r) \\
b_{n, m} \psi_{n, m}^{(1 / 2)}(r) \\
c_{n, m} \psi_{n, m}^{(-1 / 2)}(r) \\
d_{n, m} \psi_{n, m}^{(-3 / 2)}(r)
\end{array}\right)
$$

with the four spinor amplitudes given by

$$
\psi_{n, m}^{(j)}(r)=\frac{\mathcal{N}_{n, m}^{(j)}}{\ell_{\omega}[1+2(|j|-1) \bar{\gamma}]^{1 / 4}} \mathrm{e}^{-\frac{e_{j}^{2}}{2}} \varrho_{j}^{\alpha_{m}^{(j)}} \mathrm{L}_{n}^{\left(\alpha_{m}^{(j)}\right)}\left(\varrho_{j}^{2}\right)
$$

$\mathrm{L}_{n}^{(\alpha)}(x)$ is an associated Laguerre polynomial, $\mathcal{N}_{n, m}^{(j)}=$ $\left[2 \Gamma(n+1) / \Gamma\left(n+\alpha_{m}^{(j)}+1\right)\right]^{1 / 2}$ with $\Gamma(x)$ denoting the Euler Gamma function, $\varrho_{j}=r /\left(\ell_{\omega}[1+2(|j|-1) \bar{\gamma}]^{1 / 4}\right)$, and $\alpha_{m}^{(j)}=\sqrt{(m-j)^{2}+\lambda_{R}^{2} /(1+2(|j|-1) \bar{\gamma})} \cdot m$ is the eigenvalue of $\hat{M}_{z}$ and $n$ the oscillator-level index. Ansatz (5) diag- 
onalises the first term of the Hamiltonian (4b),

$$
H_{\mathrm{rg}}^{(1)}=\lambda_{d}\left(\left[n+\frac{1}{2}\right] \hat{\Gamma}^{\frac{1}{2}}+\frac{\sqrt{\hat{\Gamma}\left(m-\hat{J}_{z}\right)^{2}+\lambda_{R}^{2}}-\lambda_{R}}{2}\right) .
$$

However, the second term of $H_{\mathrm{rg}}$ in Eq. (4b is offdiagonal in spin-3/2 space, coupling $\mathrm{HH}$ and $\mathrm{LH}$ amplitudes within subspaces spanned by $\hat{J}_{z}$ eigenstates with eigenvalues $\{ \pm 3 / 2, \mp 1 / 2\}$, respectively. In addition, this term has both diagonal and off-diagonal matrix elements in the $\psi_{n, m}$ representation, i.e., it couples states with different $n$. We omit the lengthy analytical expressions for associated matrix elements. It turns out that HH-LH mixing between states having their quantum number $n$ differ by 0,1 , and 2 are most relevant. In the following, we focus on the lowest oscillator level $(n=0)$ and include only its intra-level HH-LH mixing. Neglecting the subtle difference between $\varrho_{j}$ and $\varrho_{j \pm 2}$, setting both equal to $r / \ell_{\omega}$, and replacing $\alpha_{m}^{(j)} \equiv \lambda_{R}$ yields the corresponding matrix element ${ }^{25}$

$$
\left(H_{\mathrm{rg}}^{(2)}\right)_{00}=-\frac{\lambda_{d} \bar{\gamma}}{4}\left\{\hat{J}_{+}^{2}\left[1-\frac{\left(m-\hat{J}_{z}-2\right)\left(m-\hat{J}_{z}\right)+2}{\lambda_{R}}\right]+\hat{J}_{-}^{2}\left[1-\frac{\left(m-\hat{J}_{z}+2\right)\left(m-\hat{J}_{z}\right)+2}{\lambda_{R}}\right]\right\} .
$$

Diagonalising the Hamiltonian $H_{\mathrm{qw}}+H_{\mathrm{rg}}^{(1)}+\left(H_{\mathrm{rg}}^{(2)}\right)_{00}$ yields the lowest hole-ring subband dispersions. The result is shown in Fig. 1 for a set of realistic parameters. For comparison, we also plot dispersions obtained when HH-LH mixing is neglected but HH-LH splitting taken into account, i.e., when only $H_{\mathrm{qw}}+H_{\mathrm{rg}}^{(1)}$ is considered.

Two-terminal transport and AA phase. At fixed energy $E$ between the HH-like and LH-like subband bottoms shown in Fig. 1 four propagating channels exist, having different angular momenta $m_{s \sigma}$. Here $s=+,-$ labels the two dispersion curves $(s= \pm$ corresponding to the subspace spanned by $\hat{J}_{z}$-projection eigenstates with eigenvalues $\{ \pm 3 / 2, \mp 1 / 2\}$, respectively), and $\sigma=+,-$ distinguishes opposite propagation directions. The condition for finding these angular momenta is $E_{s}\left(m_{s \sigma}\right)=E$. We now consider the following scenario, illustrated in Fig. 2. A lead attached at $\varphi=0$ is assumed to inject holes in a set of orthogonal initial states $|\nu\rangle$. Here the ket refers to a normalised spin-3/2 spinor without any dependence on spatial coordinates ${ }^{26}$ Ring eigenstates taken at $\varphi=0$ and with spatial profile neglected are given by $\chi_{s \sigma}=\left(a_{0, m_{s \sigma}}, b_{0, m_{s \sigma}}, c_{0, m_{s \sigma}}, d_{0, m_{s \sigma}}\right)$, and the injected

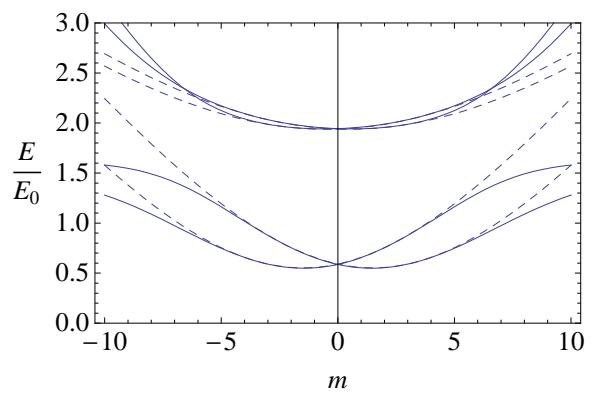

FIG. 1: Lowest hole-ring subbands (solid curves) arising from the in-plane ring-potential bound-state level with $n=0$, calculated for $\bar{\gamma}=0.37, \lambda_{d}=0.5$, and $\lambda_{R}=10$. The dashed curves are obtained when HH-LH splitting is included but HH-LH mixing is neglected. states can be written as a superposition $\mid$ in $\rangle_{\nu}=\sum_{s \sigma} \xi_{s \sigma}^{(\nu)} \chi_{s \sigma}$. Each spinor amplitude of a ring eigenstate with quantum numbers $s, \sigma$ acquires a phase during propagation around a halfring arm that is determined by its associated eigenvalue of the operator $\sigma \pi\left(\hat{M}_{z}-\hat{J}_{z}\right)$. The resulting state at $\varphi=\pi$ will be $\mid$ out $\rangle_{\nu}=\sum_{s \sigma} \xi_{s \sigma}^{(\nu)} \chi_{s \sigma} \exp \left\{\sigma i \pi\left(m_{s \sigma}-\frac{3 s}{2}\right)\right\}$. Assuming another lead being attached at $\varphi=\pi$, we find the transmission probabilities from incoming-lead channel $\nu$ to outgoing-lead channel $\mu$. Including the effect of a finite magnetic flux $\phi$ threading the area bounded by the ring, it reads

$$
T_{\nu}^{\mu}=\left|\sum_{s \sigma} \xi_{s \sigma}^{(\mu) *} \xi_{s \sigma}^{(\nu)} \mathrm{e}^{\sigma i \pi\left(m_{s \sigma}+\frac{\phi}{\phi_{0}}-\frac{3 s}{2}\right)}\right|^{2} .
$$

In Eq. (8), $m_{s \sigma} \equiv-m_{-s,-\sigma}$ are the angular-momentum eigenvalues found for $\phi=0$. The linear two-terminal ring conductance is given by $G_{\mathrm{rg}}=\frac{e^{2}}{2 \pi \hbar} \sum_{\nu, \mu} T_{\nu}^{\mu}$.

Inspection of Eq. (8) reveals the well-known signatures of the Aharonov-Bohm effect, $\underline{\underline{8}}$ arising from the interference of counter-propagating modes with conserved quantum number $s$. A phase difference $\Phi_{\mathrm{AA}}^{(s)}$ of associated quantum amplitudes will be accumulated during propagation between $\varphi=0$ and $\pi$ that is essentially an Aharonov-Anandan phase ${ }^{\frac{5}{5}}$ for holes confined in the ring. The latter can be written as the sum of a magnetic-flux-dependent part (the Aharonov-Bohm² phase

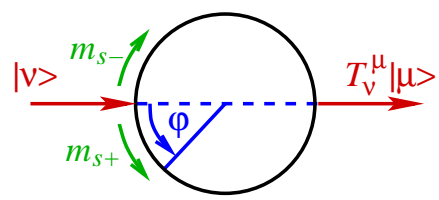

FIG. 2: (Color online) Two-terminal transport. Holes in scattering states $|\nu\rangle$ are injected via a lead attached at $\varphi=0$. Eigenmodes with angular momenta $m_{s \sigma}$ provide propagation channels in the ring. Upon reaching the point $\varphi=\pi$, interference and coupling into outgoing scattering states $|\mu\rangle$ occurs. 


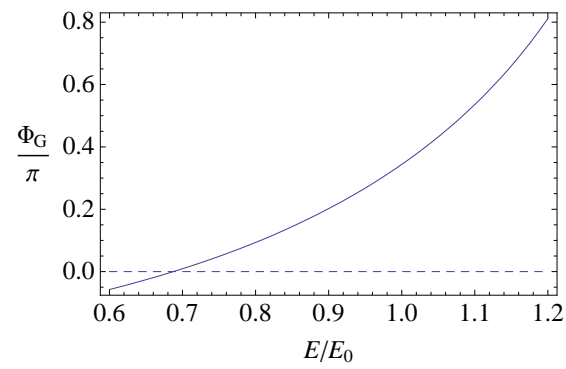

FIG. 3: Solid curve: Anomalous component $\Phi_{\mathrm{G}}$ of the AA phase appearing in the two-terminal hole-ring transmission. Parameters used in the calculation are the same as in Fig 1 Dashed curve: Corresponding result obtained when HH-LH mixing is neglected.

$\left.2 \pi \phi / \phi_{0}\right)$ and a remainder $\Phi_{\mathrm{G}}^{(s)}$ that depends on the quantum number $s$ distinguishing dispersion branches:

$$
\begin{aligned}
& \Phi_{\mathrm{AA}}^{(s)}=2 \pi \frac{\phi}{\phi_{0}}+\Phi_{\mathrm{G}}^{(s)}, \\
& \Phi_{\mathrm{G}}^{(s)}=\pi\left(m_{s+}+m_{s-}-3 s\right) .
\end{aligned}
$$

The symmetry $m_{s \sigma}=-m_{-s,-\sigma}$ implies $\Phi_{\mathrm{G}}^{(+)}=-\Phi_{\mathrm{G}}^{(-)} \equiv$
$\Phi_{\mathrm{G}}$. We plot $\Phi_{\mathrm{G}}$ for a realistic set of parameters in Fig. 3 .

Conclusions and discussion. We investigated spin-3/2 hole states confined in a quasi-one-dimensional ring. A number of controlled approximations were employed that can be systematically improved upon. We find a previously neglected energy-dependent contribution $\Phi_{\mathrm{G}}$ to the AA phase that results from HH-LH mixing and may be related to anomalous spin precession 27 of spin-3/2 particles. It is likely that this phase is the origin of numerically observed magnetooscillations of the conductance polarization in multiply connected hole nanostructures $\frac{19}{}$ that persist even in the presence of relatively strong disorder. In a magneto-conductance experiment, the value $\Phi_{\mathrm{G}}\left(E_{\mathrm{F}}\right)$ of AA phase for states at the Fermi energy would be observed as a shift in the AharonovBohm oscillations. This mimicks behaviour expected ${ }^{12.13}$ in systems with a finite zero-field (Rashba or Dresselhaus) spin splitting, which was used to interpret experimental data 16 We show that, in general, both HH-LH mixing and spin-splitting effects need to be considered. In addition, the coupling of the ring to external leads needs to be well-understood, because the character of injected hole states will depend sensitively on the lead confinement.

Acknowledgment. UZ thanks P. Brusheim and D. Csontos for useful discussions.
* Present address.

$\dagger$ Permanent address.

1 A. Shapere and F. Wilczek, eds., Geometric Phases in Physics (World Scientific, Singapore, 1989).

2 Y. Aharonov and D. Bohm, Phys. Rev. 115, 485 (1959).

3 Y. Aharonov and A. Casher, Phys. Rev. Lett. 53, 319 (1984).

${ }^{4}$ M. V. Berry, Proc. R. Soc. London, Ser. A 392, 45 (1984).

5 Y. Aharonov and J. Anandan, Phys. Rev. Lett. 58, 1593 (1987).

6 J. Anandan, J. Christian, and K. Wanelik, Am. J. Phys. 65, 180 (1997).

7 L. L. Sohn, L. P. Kouwenhoven, and G. Schön, eds., Mesoscopic Electron Transport (Kluwer, Dordrecht, 1997).

8 Y. Gefen, Y. Imry, and M. Y. Azbel, Phys. Rev. Lett. 52, 129 (1984); M. Büttiker, Y. Imry, and M. Y. Azbel, Phys. Rev. A 30, 1982 (1984).

9 R. A. Webb, S. Washburn, C. P. Umbach, and R. B. Laibowitz, Phys. Rev. Lett. 54, 2696 (1985); G. Timp, A. M. Chang, J. E. Cunningham, T. Y. Chang, P. Mankiewich, R. Behringer, and R. E. Howard, ibid. 58, 2824 (1987).

10 A. Stern, Phys. Rev. Lett. 68, 1022 (1992); D. Loss and P. M. Goldbart, Phys. Rev. B 45, 13544 (1992); A. G. Aronov and Y. B. Lyanda-Geller, Phys. Rev. Lett. 70, 343 (1993).

11 H. Mathur and A. D. Stone, Phys. Rev. Lett. 68, 2964 (1992); T.-Z. Qian and Z.-B. Su, ibid. 72, 2311 (1994).

12 J. Nitta, F. E. Meijer, and H. Takayanagi, Appl. Phys. Lett. 75, 695 (1999); D. Frustaglia and K. Richter, Phys. Rev. B 69, 235310 (2004); B. Molnar, F. M. Peeters, and P. Vasilopoulos, ibid. 69, 155335 (2004).

13 A. A. Kovalev, M. F. Borunda, T. Jungwirth, L. W. Molenkamp, and J. Sinova, Phys. Rev. B 76, 125307 (2007).

${ }^{14}$ I. Zutić, J. Fabian, and S. Das Sarma, Rev. Mod. Phys. 76, 323 (2004).

15 T. Bergsten, T. Kobayashi, Y. Sekine, and J. Nitta, Phys. Rev. Lett.
97, 196803 (2006).

16 M. König, A. Tschetschetkin, E. M. Hankiewicz, J. Sinova, V. Hock, V. Daumer, M. Schäfer, C. R. Becker, H. Buhmann, and L. W. Molenkamp, Phys. Rev. Lett. 96, 076804 (2006); B. Grbić, R. Leturcq, T. Ihn, K. Ensslin, D. Reuter, and A. D. Wieck, ibid. 99, 176803 (2007); B. Habib, E. Tutuc, and M. Shayegan, Appl. Phys. Lett. 90, 152104 (2007); N. Kang, E. Abe, Y. Hashimoto, Y. Iye, and S. Katsumoto, J. Phys. Soc. Jpn. 76, 083704 (2007).

17 R. Winkler, Spin-Orbit Coupling Effects in Two-Dimensional Electron and Hole Systems (Springer, Berlin, 2003).

$18 \mathrm{HH}$ and LH states correspond to eigenstates of spin-3/2 projection $\perp$ ring with eigenvalues $\pm 3 / 2$ and $\mp 1 / 2$, respectively.

19 J. Zhou, M. W. Wu, and M. Q. Weng, Phys. Lett. A 349, 393 (2006).

20 J. M. Luttinger, Phys. Rev. 102, 1030 (1956).

${ }^{21}$ N. O. Lipari and A. Baldereschi, Phys. Rev. Lett. 25, 1660 (1970).

${ }^{22}$ Setting $\hat{k}_{z} \rightarrow 0$ neglects a subtle renormalisation of the effective hole masses for in-plane motion. See, e.g., E. L. Ivchenko and G. E. Pikus, Superlattices and Other Heterostructures (Springer, Berlin, 1995).

23 W. Tan and J. C. Inkson, Semicond. Sci. Technol. 11, 1635 (1996).

${ }^{24}$ W. Tan and J. C. Inkson, Phys. Rev. B 60, 5626 (1999); M. Pletyukhov and V. Gritsev, ibid. 70, 165316 (2004).

25 Our approximations amount to neglecting higher-order corrections in $\bar{\gamma}$ and assuming $\lambda_{R} \gg|m|$, which is the typical regime of interest. The condition $\lambda_{d}<1$ (required for neglecting higher $2 \mathrm{D}$ quantum-well bound states) is usually satisfied.

26 The detailed form of spinor components in real space will influcence the coupling of leads to the ring. However, this is irrelevant for the AA phase, which is our current focus.

27 D. Culcer, C. Lechner, and R. Winkler, Phys. Rev. Lett. 97, 106601 (2006). 